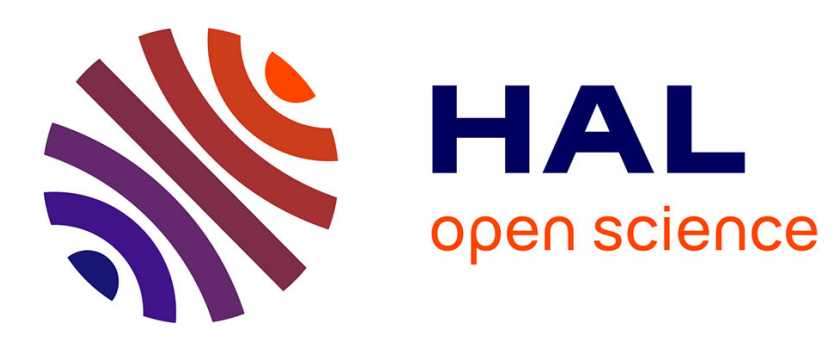

\title{
Online Intramuscular EMG Decomposition with Varying Number of Active Motor Units
}

Eric Le Carpentier, Yannick Aoustin, Jonathan Monsifrot, Dario Farina

\section{To cite this version:}

Eric Le Carpentier, Yannick Aoustin, Jonathan Monsifrot, Dario Farina. Online Intramuscular EMG Decomposition with Varying Number of Active Motor Units. International Conference on NeuroRehabilitation (Replace, Repair, Restore, Relieve - Bridging Clinical and Engineering Solutions in Neurorehabilitation), Jun 2014, Aalborg, pp.303 - 311, 10.1007/978-3-319-08072-7_50 . hal-01074903

\section{HAL Id: hal-01074903 https://hal.science/hal-01074903}

Submitted on 15 Oct 2014

HAL is a multi-disciplinary open access archive for the deposit and dissemination of scientific research documents, whether they are published or not. The documents may come from teaching and research institutions in France or abroad, or from public or private research centers.
L'archive ouverte pluridisciplinaire HAL, est destinée au dépôt et à la diffusion de documents scientifiques de niveau recherche, publiés ou non, émanant des établissements d'enseignement et de recherche français ou étrangers, des laboratoires publics ou privés. 


\title{
Online intramuscular EMG decomposition with varying number of active motor units
}

\author{
Eric Le Carpentier, Yannick Aoustin, Jonathan Monsifrot and Dario Farina
}

\begin{abstract}
This paper deals with the online decomposition of intramuscular electromyographic (iEMG) signals. A Markov model is proposed, which takes into account a varying number of firing motor neurons. A Bayes filter detects online the firing motor units by using a dictionary of approximated motor unit action potentials waveforms, and estimates precisely the action potential shapes and the respective firing rates. The method was tested on both simulated and experimental signals.
\end{abstract}

\section{INTRODUCTION}

The idea to control active prosthetic devices for amputees through surface electromyographic (EMG) signals was proposed by N. Wiener in [1]. Currently it is an active field of research, see [2]. Akazawa et al [3] estimated the force level and mechanical impedance of limb motion through EMG signals. A multiple neural network is proposed by Tsukamoto et al [4] to determine the movement intended by an amputee from surface electromyographic signals. However, most previous works were devoted to control only a particular joint depending on the torque estimated from EMG signals, see [5], [3]. There is an extreme variability in the collected signal between users and within the user himself, and, the long training of the decoding system, see [6]. The obtained movements of the prosthetic are not fluent, and precise manipulation and power grasping of heavy object is not currently possible [7]. These tasks are achieved by a combination of a large number of degrees of freedom. Current commercial prosthetic hands are unable to provide enough grasping or manipulation functionality. However, recent works promote the use of intramuscular EMG (iEMG) signals [8] to reach this objective.

We believe that the information on the activity of individual motor neurons will help to improve the control of prosthetic hands, although this approach is more challenging than classic methods that use the EMG as a compound signal. Electromyographic (EMG) signals represent the activity of muscle fibers, as driven by the population of spinal motor neurons (MN) innervating the muscle. Thus, despite being measured peripherally, EMG signals reflect neural activity since they contain information on the activation drive sent from the spinal cord to the muscles (neural drive to the muscles). The identification of individual MN spikes from the EMG signal is called EMG decomposition

E. Le Carpentier ${ }^{\dagger}$, Y. Aoustin ${ }^{\ddagger}$ and J. Monsifrot ${ }^{\ddagger}$ are with LUNAM Université ( ${ }^{\dagger}$ Ecole Centrale de Nantes, ${ }^{\ddagger}$ Université de Nantes) and IRCCyN (UMR CNRS 6597), 1, rue de la Noë, 44321 Nantes, France (e-mail: (Eric.Le-Carpentier|Yannick.Aoustin|Jonathan.Monsifrot)@irccyn.ecnantes.fr).

D. Farina is with the Department of Neurorehabilitation Engineering, Bernstein Center for Computational Neuroscience, University Medical Center Göttingen Georg-August University, Von-Siebold-Str. 4, 37075 Göttingen, Germany, (e-mail: dario.farina@bccn.uni-goettingen.de).
[9]. To allow physiological investigations of motor unit (MU) behavior during muscle contractions, decomposition methods for (iEMG), have been developed, see [10], [11], [12], or [13]. Through theses investigations applied offline, including the interaction of an expert operator to optimize the accuracy of the result, the neural strategies for movement control are decoded. In [14] a decomposition method, which could be potentially implemented online, is proposed. It is based on a new sequential algorithm for the EMG decomposition that allows full decomposition in a sequential way. This method processes a singlechannel iEMG signal sequentially. The iEMG signals are modeled as a sum of independent convolved spike trains. The recording of a whole sequence of spike trains is not necessary to compute the estimates of the parameters of the model. The sparsity and the regularity of the spike trains is taken into account by a stochastic model for the time between two spikes of the same train, based on a discrete Weibull distribution [15]. Then, an online estimation method for the parameters of the Weibull distribution and of the motor unit action potentials (MUAP) shapes is implemented.

This paper extends the original contribution [14], in which the number of firing motor neurons was supposed to be fixed and known.

\section{METHODS}

\section{A. Basic hypotheses}

An iEMG signal is an electrical signature of muscle activity, i.e. activity of muscle fibers. As remarked in [16], [17], the property of the linearity of the sum of electrical signals justifies modeling the observed signal $Y$ as a sum of filtered spike trains embedded in a noise $W$; for all discrete time indexes $n$ :

$$
Y[n]=\sum_{i=1}^{n_{\mathrm{M}}[n]}\left(h_{i} * U_{i}\right)[n]+W[n]
$$

For each source, a time-invariant linear filtering effect is considered. It appears as a convolution between the input spike train $U_{i}$ and an impulse response $h_{i}$ :

- the spike trains $U_{i}$ are sparse 0-1 processes which firing rates are linked to the muscle activity;

- the shapes of the impulse responses $h_{i}$ represent the MUAP and are assumed time-invariant.

In a probabilistic framework, the spike train sequences and the noise sequence are stochastic processes. They are supposed mutually independent. The noise is assumed independent along time, zero-mean, gaussian, with constant variance $v$. The spike trains $U_{i}$ are not available. The impulse responses $h_{i}$ are assumed of finite length 
with known maximum length $\ell_{\mathrm{IR}}$. The noise variance $v$ is unknown. A dictionary of rough shapes of all possible wavelets $h_{i}$ is necessary, but the $n_{\mathrm{M}}[n]$ motor neurons for which discrete time $n$ belongs to a firing epoch are unknown.

From this convolution model, we aim to derive an hidden Markov model (HMM), whose state will be representative of the process: active motor neurons, MUAP shapes, firing rates. Then, Bayesian filtering will estimate online the model state.

\section{B. Hidden Markov model}

For each input source $U_{i}$, in a firing epoch, interspike intervals $\Delta_{i}[k]$ - discrete time length between spikes, $k \in$ $\mathbb{Z}$ being the spike index - are supposed to be independent and identically distributed (i.i.d.) random variables, with a parameterized probability mass function (PMF) defined by $\operatorname{Pr}\left(\Delta_{i}[k]=t \mid \mathbf{\Theta}_{i}\right)$, for all positive natural numbers $t \geq 1$, where $\boldsymbol{\Theta}_{i}$ is an unknown parameter vector (which will be considered as random in the Bayesian framework below).

For each source, we introduce the sawtooth sequence $T_{i}[n]$, which corresponds to the time interval since the last spike. The value $T_{i}[n]$ is incremented at each time index, unless the MN fires; it is then set to zero:

$$
T_{i}[n+1]= \begin{cases}0 & \text { if the MN fires at time } n+1 \\ T_{i}[n]+1 & \text { otherwise }\end{cases}
$$

By means of the Kronecker delta, the spike trains write:

$$
U_{i}[n]=\delta\left(T_{i}[n]\right)
$$

Let us use the exponent ${ }^{n}$ to denote a time span till time $n$ (e.g. $\left.T_{i}^{n}=\left(T_{i}[j]\right)_{1 \leq j \leq n}\right)$. Then, under the assumption that the sequence of interspike intervals is i.i.d., it is shown in [14] that the sequence $T_{i}[n]$ is Markovian; for all possible time spans $t$ (positive integer valued) since the last spike:

$$
\operatorname{Pr}\left(T_{i}[n+1]=t \mid T_{i}^{n}, \boldsymbol{\Theta}_{i}\right)=\operatorname{Pr}\left(T_{i}[n+1]=t \mid T_{i}[n], \boldsymbol{\Theta}_{i}\right)
$$

As shown in [14], choosing a discrete Weibull distribution for the interspike interval (with 2 parameters: a location one, and a concentration one), this transition probability and the firing rate (that is $1 / E\left\{\Delta_{i}[k] \mid \Theta_{i}\right\}$ ) are easy to calculate.

The state of the hidden Markov model contains the set of the indexes of active motor neurons picked in the dictionary of all possible MNs (sources), with their associated sojourn times and Weibull parameters, that is, for all $n \geq 1$ :

- $\mathbf{A}[n]$, the set of the $n_{\mathrm{M}}[n]$ active sources;

- $\mathbf{T}[n]=\left[T_{i}[n]\right]_{i \in \mathbf{A}[n]}$, concatenation of the $n_{\mathrm{M}}[n]$ sojourn times (column vector with value in $\mathbb{N}^{n_{\mathrm{M}}[n]}$ );

- $\boldsymbol{\Theta}[n]$, concatenation of the $n_{\mathrm{M}}[n]$ parameters $\boldsymbol{\Theta}_{i}$ of the Weibull distributions (column vector with value in $\mathbb{R}^{2 n_{\mathrm{M}}[n]}$;

- $\mathrm{H}[n]$, concatenation of the coefficients of the MUAP (column vector with value in $\mathbb{N}^{\ell_{\mathrm{I}} n_{\mathrm{M}}[n]}$ );

Note that the size of the state is time-varying. The set $\overline{\mathbf{A}}[n]$ is the set of idle motor neurons indexes in the dictionary.

The transition law from time $n$ to time $n+1$ is built as a birth and death process, with the help of 2 tuning parameters $t_{\mathrm{I}}$ and $\lambda$ :
- if some sojourn times reach a maximum time $t_{\mathrm{I}}$, the corresponding motor neuron indexes are dropped from $\mathbf{A}[n+1]$

- otherwise, an inactive motor unit is picked at random in the dictionary and added in $\mathbf{A}[n+1]$ with probability $\lambda$ (that is to say, there is no activation with probability $1-\lambda$ ).

The Weibull parameters and the MUAP shapes are assumed constant, the sojourn time evolves thanks to transition (2), if the corresponding motor neuron is kept in the transition above.

The observation equation, which is equivalent to the representation (1), becomes:

$$
Y[n]=\underbrace{\left[\boldsymbol{\varphi}_{T_{1}[n]} \cdots \boldsymbol{\varphi}_{T_{n_{\mathrm{M}}}[n]}\right]}_{\boldsymbol{\psi}_{\mathbf{T}[n]}} \mathbf{H}+W[n]
$$

where $\varphi_{t}$ is a row vector of size $\ell_{\mathrm{IR}}$ that all components are 0 , except, if $t<\ell_{\mathrm{IR}}$, the component in position $t+1$ that has value 1 .

\section{Bayes filtering}

The purpose of a Bayes filter is to propagate along time the posterior probability law of the state sequence of a HMM. Using calculations similar to those found in [14], we can derive recursions:

- on posterior probability density functions of continuous-valued variable $\mathrm{H}[n]$, given $\mathbf{A}[n]$ and $\mathbf{T}[n]$; note that this is nothing but a rigorous implementation of the well known Kalman filter;

- on posterior probability density functions of continuous-valued variable $\boldsymbol{\Theta}[n]$, given $\mathbf{A}[n]$ and $\mathbf{T}[n]$, by means of a reasonable approximation;

- on posterior probability of all possible values of discrete-valued variables $(\mathbf{A}[n], \mathbf{T}[n])$.

At each step $n$, the paths $(\mathbf{A}[n], \mathbf{T}[n])$ are extended thanks to the birth and death process described above. Obviously, it is impossible to process all possible values of $(\mathbf{A}[n], \mathbf{T}[n])$, since their number increases exponentially as time index $n$ grows. Only the $n_{\text {path }}$ most probable sequences will be kept, the number $n_{\text {path }}$ being a parameter of the method similar to the number of particles in a particle filter [18].

For all MNs in the dictionary, the MUAP shape in the dictionary is corrected using the Minimum Mean Square Error (MMSE) estimate obtained as a weighted mean value among the $n_{\text {path }}$ state estimators linked to a path containing this motor neuron index (the weight being the posterior probability of the path). The same principle is used to obtain the current estimation of the Weibull parameters.

Note that a forgetting factor is implemented to track slow variations in MUAP shapes and Weibull parameters, so that the filter becomes adaptive. This forgetting factor can be interpreted as an equivalent window length (see [19], [14]).

\section{Simulated signals and experimental signal}

Simulated signals were generated with the Markov model. A $10 \mathrm{kHz}$ sampling frequency was assumed for the simulated signals to ease comparison with the experimental signal. 
The filters shapes were obtained from experimental iEMG signals to make the simulation more realistic. The signal to noise ratio (SNR) was set to $11 \mathrm{~dB}$. Motor neurons were sequentially activated, then deactivated. Although this simulation does not reflect a precise physiological mechanism, it allows to test, over a single trial, the tracking capability of the algorithm over time-varying parameters, as well as its capability of locking the values of parameters constant over time.

The experimental iEMG signals were recorded from the extensor digitorum muscle of a healthy subject (age 24 years), with a pair of wire electrodes made of Teflon coated stainless steel (A-M Systems, Carlsborg, WA, USA; diameter $50 \mu \mathrm{m}$ ) inserted into the muscle belly with a 25 $\mathrm{G}$ needle, with insertion at approximately 45 degrees with respect to the skin plane. High selectivity of the intramuscular recording system was obtained by cutting the wires to expose only the cross-section (recording surface). The needle was then removed with the fine wires left into the muscle for the recording. The muscle selected corresponds to a source of EMG activity often used for the control of prostheses by transradial amputees. The iEMG signals were amplified bipolarly (Counterpoint EMG, DANTEC Medical Skovlunde, Denmark), band-pass filtered (500 Hz$5 \mathrm{kHz}$ ), and sampled at $10 \mathrm{kHz}$. During the experiment, the subject had access to a visual feedback of the exerted force. He performed few changes in the contraction force during the experiment. A manual processing produced 5 different shapes to feed the initial dictionary.

Algorithm was applied to both the simulated signals and the experimental one, with adaptivity corresponding to a one-second equivalent window length. The number of selected paths $n_{\text {path }}$ was set to 64 .

\section{RESULTS}

Results on simulated data (figure 1) did not reveal any missed spikes, and discharge rates were correctly estimated. Activations and deactivations were correctly detected, although a delay can be observed at deactivation, which could be minimized by means of a smaller $t_{\mathrm{I}}$ parameter. Note that the difference between simulated signal and noise-free reconstruction almost perfectly rebuilds the original additive white noise.

Results on iEMG data (figure 2) were not compared to those of an expert, but, despite a few glitches, one can see that the automatically detected switches in the number of active motor neurons (between 0 and 5) provide a low mean square error between actual signal and noisefree reconstruction. The figure shows the regularity of the MUAP trains; this regularity is favored by the stochastic model that drives the interspike intervals.

\section{PERSPECTIVES}

This paper proposes a new decomposition algorithm of iEMG signals which tackles both the problems of time-varying discharge rates and time-varying activation of motor neurons. He has an online architecture which makes it usable in applications which need on-the-fly estimation, such as prosthesis control. Nevertheless, practical implementation remains to be done: the algorithm has a parallel architecture ( $n_{\text {path }}$ scenarios are played together) which could be implemented using parallel computating, e.g. GPGPU (General-Purpose computing on Graphics Processing Units). For prosthesis control, the mapping between iEMG decomposition and actual forces and movements remains to be done. Automatic adjustment of the equivalent window length has to be investigated: this length can be high for precise and meticulous tasks, but should be low for fast and nervous movements.

\section{REFERENCES}

[1] N. Wiener, CYBERNETICS or control and communication in he animal and the machine. MIT Press, 1948.

[2] C. Castellini, P. van der Smagt, G. Sandini, and G. Hirzinger, "Surface EMG for force control of mechanical hands," in IEEE Int. Conf. on Robotics and Automation, 2008., May 2008, pp. 725-730.

[3] K. Akazawa, H. Takizawa, K. Hayashi, and K. Fujii, "Development of control system and myoelectric signal processor for bio-mimetic prosthetic hand," Biomechanism 9, vol. 19, no. 4, pp. 43 - 53, 1988

[4] M. Tsukamoto, T. Kondo, and K. Ito, "A prosthetic hand control based on non stationary emg at the start of movement," $J$. of Robotics and Mechatronics, vol. 19, no. 4, pp. 382 - 134, 2007.

[5] C. J. Abul-haj and N. Hogan, "Functional assessment of control systems for cybernetic elbow prostheses-part i, part ii," IEEE Trans. Biomed. Engineering, vol. 37, no. 11, pp. 1025 - 1047, 1990.

[6] K. Englehart, B. Hudgins, P. Parker, and M. Stevenson, "Classification of the myoelectric signal using time-frequency based representations," Medical Engineering and Physics, vol. 21, pp 431-438, 1999.

[7] M. Zecca, S. Micera, M. C. Carrozza, and P. Dario, "Control of multifunctional prosthetic hands by processing the electromyographic signal," Critical Reviews in Biomedical Engineering, vol. 30, no. 4-6, pp. 459 - 485, 2002.

[8] E. N. Kamavuako, K. B. Englehart, W. Jensen, and D. Farina, "Simultaneous and Proportional Force Estimation in Multiple Degrees of Freedom From Intramuscular EMG." IEEE Trans. Biomed. Engineering, vol. 59, no. 7, pp. 1804-1807, 2012.

[9] B. Mambrito and C. De Luca, "A Technique for the Detection, Decomposition and Analysis of the EMG Signal," Electroencephalography and Clinical Neurophysiology, pp. 58:175-188, 1984.

[10] R. Lefever and C. De Luca, "A Procedure for Decomposing the Myoelectric Signal Into Its Constituent Action Potentials - Part I: Technique, Theory, and Implementation," IEEE Trans. Biomed. Engineering, vol. 29, no. 3, pp. 149-157, Mar. 1982.

[11] _ _ "A Procedure for Decomposing the Myoelectric Signal Into Its Constituent Action Potentials - Part II: Execution and Test for Accuracy," IEEE Trans. Biomed. Engineering, vol. 29, no. 3, pp. 158-164, Mar. 1982

[12] H. Marateb, S. Muceli, K. Mcgill, R. Merletti, and D. Farina, "Robust decomposition of single-channel intramuscular EMG signals at low force levels," IEEE Trans. on Automatic Control, vol. 8, no. 6, pp. 1-13, 2011.

[13] D. Ge, E. Le Carpentier, and D. Farina, "Unsupervised Bayesian Decomposition of Multiunit EMG Recordings Using Tabu Search," IEEE Trans. Biomed. Engineering, vol. 57, no. 3, pp. 561-570, 2010.

[14] J. Monsifrot, E. Le Carpentier, Y. Aoustin, and D. Farina, "Sequential Decoding of Intramuscular EMG Signals via Estimation of a Markov Model," IEEE Transactions on Neural Systems and Rehabilitation Engineering, 2014, to appear.

[15] T. Nakagawa and S. Osaki, "The discrete Weibull distribution," IEEE Trans. on Reliability, vol. R-24, no. 5, pp. 300-301, Dec. 1975.

[16] D. Stashuk, "EMG signal decomposition: how can it be accomplished and used?" J. of Electromyography and Kinesiology, vol. 11, no. 3, pp. 151-173, 2001

[17] D. Farina, A. Crosetti, and R. Merletti, "A model for the generation of synthetic intramuscular EMG signals to test decomposition algorithms," IEEE Trans. Biomed. Engineering, vol. 48, no. 1, pp. 66-77, Jan. 2001.

[18] A. Doucet, N. De Freitas, and N. Gordon, Sequential Monte Carlo methods in practice. Springer, 2001.

[19] L. Ljung and T. Söderström, Theory and Practice of Recursive Identification. Massachusetts and London: The MIT Press, 1983. 

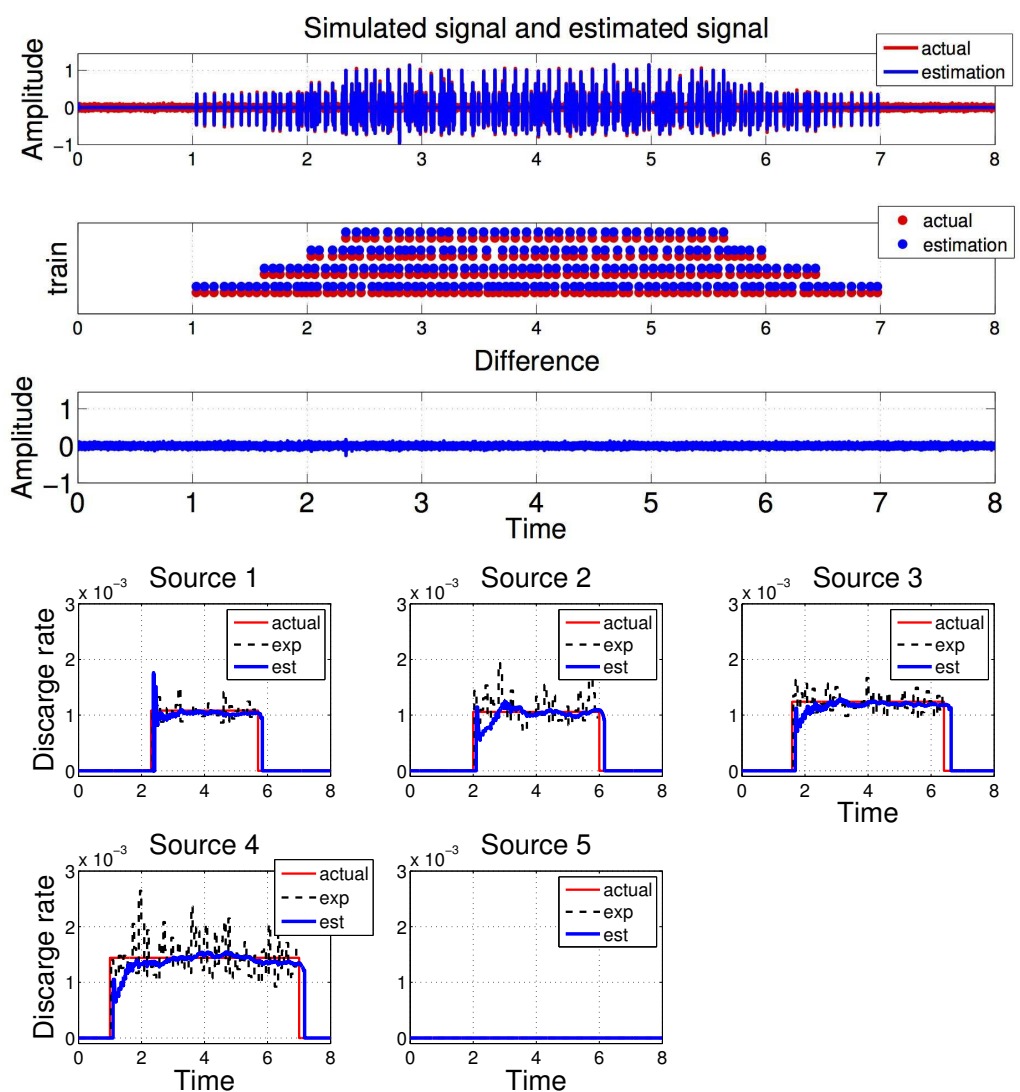

Figure 1: Simulations: actual signal and reconstructed one, actual trains and estimated ones, actual firing rates and estimated ones
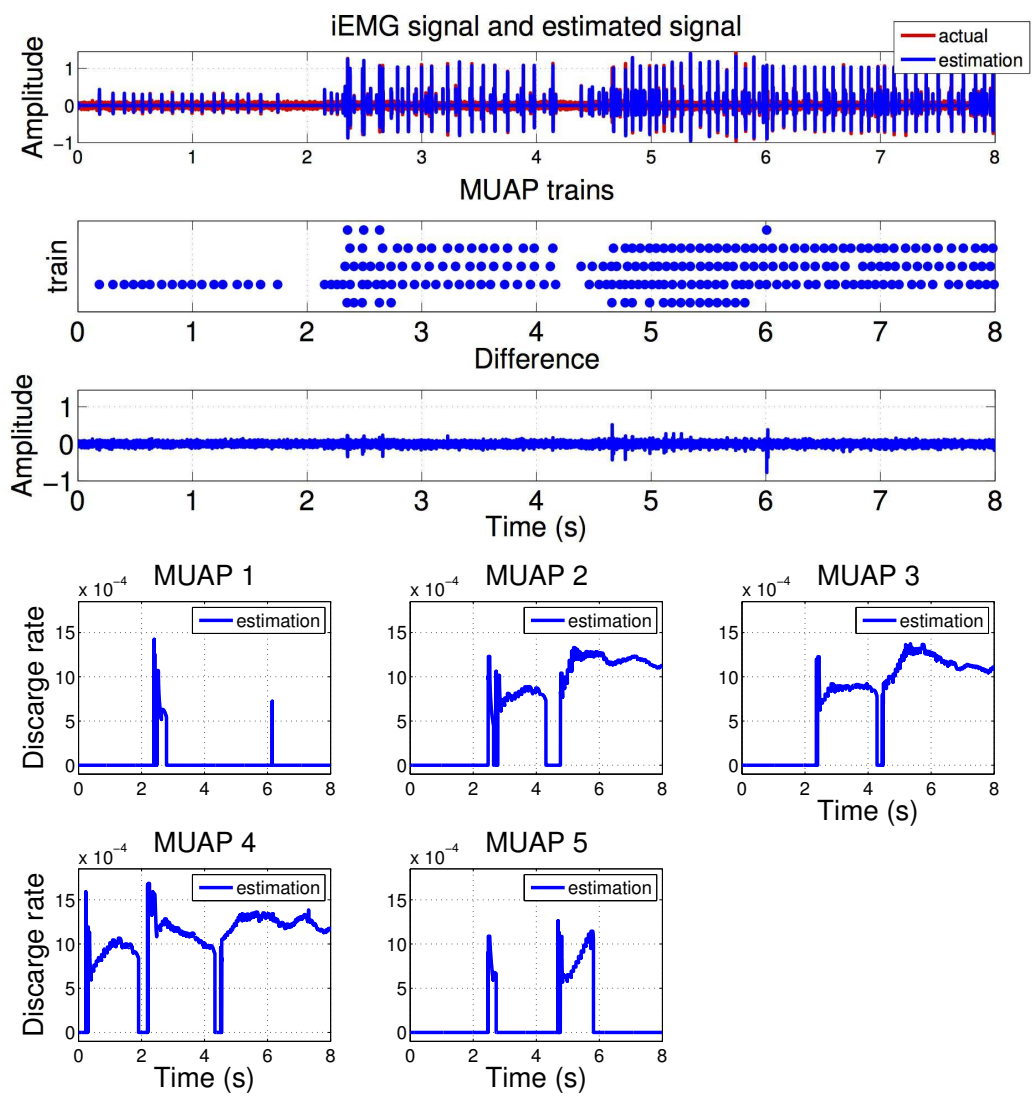

Figure 2: iEMG: actual signal and reconstructed one, estimated trains, estimated firing rates 